\title{
Spin-density and charge-density excitations in quantum wires
}

\author{
Arne Brataas ${ }^{1}$ and A. G. Mal'shukov ${ }^{2}$ and Christoph Steinebach ${ }^{3}$ and \\ Vidar Gudmundsson ${ }^{4}$ and K. A. Chao ${ }^{1}$ \\ 1 Department of Physics, Norwegian University of Science and Technology, \\ N-7034 Trondheim, Norway. \\ ${ }^{2}$ Institute of Spectroscopy, Russian Academy of Sciences, \\ 142092 Troitsk, Moscow Region, Russia. \\ 3 Institut für Angewandte Physik, Jungiusstraße 11, \\ D-20355 Hamburg, Federal Republic of Germany. \\ 4 Science Institute, University of Iceland, Dunhaga 3, IS-107 Reykjavik, Iceland.
}

(October 15, 2018)

\begin{abstract}
We study an interacting electron gas in a quantum wire within the HartreeFock random phase approximation. Vertex corrections to the electron spin polarizability due to the electronic exchange interaction are important giving rise to spin-density excitations (SDE) with large oscillator strength shifted to lower energies with respect to single-particle states. The energy of intersubband SDE oscillates with the number of subbands occupied and has a minimum when a subband energy is close to the chemical potential. Intrasubband SDE have a linear dispersion at small wave-vectors. The corresponding sound velocity is reduced with respect to the Fermi velocity due to exchange interaction within the occupied subbands and exchange screening caused by virtual transitions to upper subbands. For intersubband and intrasubband charge-density excitations (CDE) vertex corrections are of less importance. For only a single subband occupied the screening of CDE and SDE in the
\end{abstract}


Tomonaga-Luttinger model due to virtual transitions to upper subband is studied, where the virtual transitions are treated within the Hartree-Fock approximation. The calculations are in good qualitative agreement with experiments.

78.30.Fs,78.66.Fd,73.20Mf

Typeset using REVTEX 


\section{INTRODUCTION}

In recent years progress has been made in preparation and spectroscopic measurements of semiconductor nanostructures where electrons are free to move in only one direction. The parent system in fabrication of such structures is a doped two-dimensional quantum well with large $(\sim 20 \mathrm{meV})$ separation between subbands, so that electrons reside in the lower subbands. By applying a gate voltage it is possible to further confine the two-dimensional electron gas into a one-dimensional wire. The confinement gives rise to one-dimensional subbands in the single-particle electron energy spectrum with a subband separation of several meV. This is a very clean system to study since the mean free path and inelastic scattering lengths may be longer than the length of the wire which is typically of the order of some $\mu \mathrm{m}$. As a result interesting quantum effects may appear. At one-dimensional electron density about $10^{6} \mathrm{~cm}^{-1}$ more than one subband can be occupied.

In Raman spectrat I collective spin and charge density excitations of GaAs quantum wires were observed. The dispersion of low-frequency intrasubband SDE and CDE (plasmons) is clearly seen in angular resolved spectra.2 3 The wave-vector dependence of the spin-wave energy was found to be linear. The corresponding sound velocity is an important parameter which depends on the strength of the exchange interaction between electrons. Theoretical calculations based on the Hartree random-phase approximation 6 - have shown good qualitative and quantitative agreement with experiments for the intrasubband and intersubband charge-density excitations.

The role of the exchange interaction is crucial for the collective intrasubband and intersubband SDE. The direct long-range Coulomb interaction leads to depolarization shifts of single-particle excitations (SPE) and to the appearance of collective plasma modes. Similarly the exchange interaction gives rise to red shifts of spin-flip excitation energies. As a result, collective SDE with a large oscillator strength split off the continuum of SPE. This has been shown in Hartree-Fock RPA calculations in quantum well semiconductor structures 10 - 13 In this paper we present analysis of electron excitations in a GaAs quantum wire within the 
time dependent Hartree-Fock approximation. The confinement potential was chosen to be parabolic. We consider a wire of a finite length much larger than the electron localization length for electrons confined in a parabolic well. The properties of the system have been calculated for a wide range of electron numbers and occupied subbands.

We are here primarily interested in the collective spin-density and charge-density excitations that are two-particle processes. The Hartree-Fock approximation is well suited to describe the high energy excitations and all virtual intersubband transitions. The HF-RPA equations are treated totally consistently in the numerical approach, i.e. by first solving for the HF single-particle states and then with this HF basis solving the equation for the twoparticle propagator including vertex corrections. This is important in order not to violate conservation laws, e.g. to satisfy the generalized Kohn's theorem for dipole intersubband charge-density excitations. In the dipole approximation, intersubband charge-density excitations at $q=0$ are simply given by the confinement energy since the perturbation only couples to the center of mass motion which is decoupled from the relative motion in a parabolic confinement 14 Therefore the intersubband charge-density excitations at $q=0$ do not provide much information about the system. However the intersubband spin-density excitations depend on the relative motion of electrons with different spin orientation and their energies are not given simply by the confinement energy. Indeed we found SDE energies to be dependent on the electron density. These energies oscillate with the occupation of subbands and have a minimum when a new subband starts to be filled. For large wave vectors the Landau damping becomes significant for the intrasubband charge-density and spin-density excitations.

The HF-RPA, being a more or less reasonable tool for studying intersubband excitations, is not a good approach for low energy intrasubband SDE and CDE in quantum wires with one or only few subbands occupied. As known 15 even weak interactions between electrons in one-dimensional systems change their ground state from the Fermi to the Luttinger liquid. On the other hand, it was shown 16.17 that the Hartree random phase approximation gave the same dispersion for the low-energy plasmons in the spinless system as the Tomonaga- 
Luttinger model. One can not expect that in a similar way HF-RPA gives correct results for the SDE spectrum at small wave vectors when electron spins are taken into account. The Tomonaga-Luttinger model (TLM) $15,18-22$ is more appropriate for this purpose. However, this model is restricted to essentially low-energy physics and cannot be applied to quasione-dimensional systems where the coupling between the subbands is siginficant. Even if only one subband is occupied electron virtual transitions to higher nonoccupied subbands should be taken into account. These transitions cause the direct and exchange intersubband screening which renormalizes the intrasubband excitation energies. The HF-RPA can be used for evaluation of these screening effects. We thus combine the two approaches and treat the low-energy intrasubband excitations in the Tomonaga-Luttinger model, while their intersubband screening was calculated with the use of HF-RPA. We calculate the direct and exchange screening as a function of the confinement energy. It is shown that the screening effects are rather weak, stronger for SDE than for CDE and of the order of the correction due the backscattering caused by exchange scattering from one side of the Fermi surface to the other for sufficiently small subband separation $(\sim 5 \mathrm{meV})$. In an experimental situation, these effects may be just as important as the backscattering effect and they are not easy to separate. The screening may be varied by changing the parabolic confinement, but keeping the electronic density constant.

The paper is organized in the following way. Section 【 describes the model treating the ground state and excited states within the Hartree-Fock random phase approximation. Our results for the intersubband excitations are presented in section [II]. The intrasubband excitations are discussed in section IV including the calculation of the intersubband screening factors within the Tomonaga-Luttinger model. In addition the appendices show detailed evaluations of some crucial Coulomb matrix elements and how the excitation energies in the system can be found from a symmetric eigenvalue problem in the HF-RPA. 


\section{MODEL}

We consider a strictly two-dimensional electron system lying in the $x-y$ plane. The motion in the $z$ direction is neglected since we assume that the actual thickness of the well is smaller than all other relevant length scales. We use the Hartree-Fock approximation to reduce the many-particle Hamiltonian to a single-particle Hamiltonian for each electron in an effective potential approximating the electron-electron interaction. The corresponding Hartree-Fock single-particle propagator is shown in Fig. 11. For the charge-density and spin-density correlation functions we use the corresponding time-dependent approximation describing the self-consistent linear response of the two-dimensional electron gas to an external perturbation (Hartree-Fock random phase approximation). We assume that the ground state is paramagnetic. The Hartree-Fock equation for a quasi-particle is then

$$
\begin{aligned}
& {\left[-\frac{\hbar^{2}}{2 m^{*}} \nabla^{2}+V_{\mathrm{c}}(y)+2 \frac{e^{2}}{\kappa} \int d \boldsymbol{r}^{\prime} \sum_{b} f_{b} \frac{\psi_{b}^{*}\left(\boldsymbol{r}^{\prime}\right) \psi_{b}\left(\boldsymbol{r}^{\prime}\right)}{\left|\boldsymbol{r}-\boldsymbol{r}^{\prime}\right|}\right] \psi_{a}(\boldsymbol{r})} \\
& -\frac{e^{2}}{\kappa} \int d \boldsymbol{r}^{\prime} \sum_{b} f_{b} \frac{\psi_{b}^{*}\left(\boldsymbol{r}^{\prime}\right) \psi_{b}(\boldsymbol{r})}{\left|\boldsymbol{r}-\boldsymbol{r}^{\prime}\right|} \psi_{a}\left(\boldsymbol{r}^{\prime}\right)=\epsilon_{a} \psi_{a}(\boldsymbol{r})
\end{aligned}
$$

where $f_{a}$ is the Fermi occupation factor for a state with orbital quantum number $a$ (the spin is up or down) with eigenenergy $\epsilon_{a}$ and $m^{*}$ is the effective mass. The dielectric constant of the surrounding medium is noted by $\kappa$. We assume that the confining potential is parabolic

$$
V_{c}(y)=\frac{1}{2} m^{*} \omega_{0}^{2} y^{2}
$$

giving a subband spacing of $\hbar \omega_{0}$. Periodic boundary condition in the longitudinal direction of the wire gives a Bloch-type single-particle wave function

$$
\Psi_{n k}(x, y)=\frac{1}{\sqrt{L_{x}}} e^{i k x} \psi_{n k}(y)
$$

where $n$ is the subband index and the longitudinal wave-vector is $k=p \cdot 2 \pi / L_{x}$ with $p \in Z$ and $L_{x}$ is the length of the wire. For noninteracting electrons in a simple one-dimensional parabolic potential the transverse single-particle eigenfunctions are independent of the longitudinal wave-vector 


$$
\phi_{n}(y)=\frac{1}{\sqrt{l_{0}}} \frac{1}{\sqrt{2^{n} n ! \sqrt{\pi}}} H_{n}\left(\frac{y}{l_{0}}\right) \exp \left[-\frac{y^{2}}{2 l_{0}^{2}}\right],
$$

where the $n$th Hermite polynomial is denoted by $H_{n}$. The electron is localized in the transverse direction within the order of the confinement length defined as $l_{0}=\left[\hbar /\left(m^{*} \omega_{0}\right)\right]^{1 / 2}$. The eigenenergies corresponding to the eigenstates (4) are

$$
E_{n k}=\hbar \omega_{0}\left(n+\frac{1}{2}\right)+\frac{\hbar^{2} k^{2}}{2 m^{*}}
$$

The effective single-particle Hamiltonian corresponding to (11) is diagonalized using the wave functions of the noninteracting electrons (4) as a functional basis and the self-consistent solutions are obtained by iteration.

For inelastic light scattering not close to resonance the Raman intensities in polarized and depolarized scattering geometries are proportional to the imaginary parts of the chargedensity correlation function and spin-density correlation function respectively.23 The chargedensity correlation function is

$$
\chi^{+}(\boldsymbol{q}, \omega)=-\frac{i}{\hbar} \int_{0}^{\infty} d t e^{i \omega t}\langle|[\hat{\rho}(\boldsymbol{q}, t), \hat{\rho}(-\boldsymbol{q}, 0)]|\rangle,
$$

and the spin-density correlation function is

$$
\chi^{-}(\boldsymbol{q}, \omega)=-\frac{i}{\hbar} \int_{0}^{\infty} d t e^{i \omega t}\langle|[\hat{\sigma}(\boldsymbol{q}, t), \hat{\sigma}(-\boldsymbol{q}, 0)]|\rangle
$$

where $\hat{\rho}(\boldsymbol{q}, t)$ is the Fourier transform of the charge-density operator and $\hat{\sigma}(\boldsymbol{q}, t)$ is the Fourier transform of the spin-density operator along the spin quantization axis and $\langle|\ldots|\rangle$ denotes the thermodynamic average. In fact there are three different possible spin-density correlation functions, however this triplet is degenerate for the nonmagnetic system discussed here. We denote the real part of the correlation functions $(\sqrt[6]{6})$ and $\left([\overline{0})\right.$ as $R^{ \pm}(\boldsymbol{q}, \omega)$ and the imaginary part as $S^{ \pm}(\boldsymbol{q}, \omega)$

$$
\chi^{ \pm}(\boldsymbol{q}, \omega)=-R^{ \pm}(\boldsymbol{q}, \omega)-i S^{ \pm}(\boldsymbol{q}, \omega)
$$

The real and imaginary parts of retarded correlation functions are related by the KramersKronig relations which is used in the numerical evaluation of the real part of the correlation function, see Appendix B. 
If treated exactly the spectral function of the charge-density and spin-density correlation function should satisfy the longitudinal f-sum rule independent of the Coulomb interaction

$$
\int_{-\infty}^{\infty} d \omega \omega S^{ \pm}(\boldsymbol{q}, \omega)=\pi \frac{q^{2}}{m^{*}} N
$$

where $N$ is the number of electrons in the wire. The charge-density operator is

$$
\hat{\rho}(\boldsymbol{q})=\sum_{a, b} \int d \boldsymbol{r} e^{i \boldsymbol{q} \cdot \boldsymbol{r}} \psi_{a}^{*}(\boldsymbol{r}) \psi_{b}(\boldsymbol{r})\left(\hat{c}_{a \uparrow}^{\dagger} \hat{c}_{b \uparrow}+\hat{c}_{a \downarrow}^{\dagger} \hat{c}_{b \downarrow}\right)
$$

and the spin-density operator is

$$
\hat{\sigma}(\boldsymbol{q})=\sum_{a, b} \int d \boldsymbol{r} e^{i \boldsymbol{q} \cdot \boldsymbol{r}} \psi_{a}^{*}(\boldsymbol{r}) \psi_{b}(\boldsymbol{r})\left(\hat{c}_{a \uparrow}^{\dagger} \hat{c}_{b \uparrow}-\hat{c}_{a \downarrow}^{\dagger} \hat{c}_{b \downarrow}\right)
$$

expressed in terms of quasi-particle Hartree-Fock states where $\hat{c}_{a \uparrow}$ destroys a particle in a state with orbital quantum number $a$ and spin up. Therefore the correlation functions (6) and (7) may be written as

$$
\chi^{ \pm}(\boldsymbol{q}, \omega)=\sum_{a b c d}\left\langle a\left|e^{i \boldsymbol{q} \cdot \boldsymbol{r}}\right| b\right\rangle\left\langle c\left|e^{-i \boldsymbol{q} \cdot \boldsymbol{r}}\right| d\right\rangle \Pi_{a b ; c d}^{ \pm}(\omega)
$$

where the two-particle charge-density and spin-density correlation functions

$$
\begin{aligned}
\Pi_{a b ; c d}^{ \pm}(\omega)= & \Pi_{a \uparrow b \uparrow ; c \uparrow d \uparrow}(\omega) \pm \Pi_{a \uparrow b \uparrow ; c \downarrow d \downarrow}(\omega) \pm \\
& \Pi_{a \downarrow b \downarrow ; c \uparrow d \uparrow}(\omega)+\Pi_{a \downarrow b \downarrow ; c \downarrow d \downarrow}(\omega)
\end{aligned}
$$

are combinations of the retarded two-particle Green's function defined as

$$
\begin{aligned}
& \Pi_{\alpha \beta ; \gamma \delta}(\omega)= \\
& -\frac{i}{\hbar} \int_{0}^{\infty} d t e^{i \omega t}\left\langle\left|\left[\hat{c}_{\alpha}^{\dagger}(t) \hat{c}_{\beta}(t), \hat{c}_{\gamma}^{\dagger}(0) \hat{c}_{\delta}(0)\right]\right|\right\rangle .
\end{aligned}
$$

Here greek indices contain orbital and spin quantum numbers. The two-particle Green's function (14) are found in the HF-RPA, which neglects correlation effects. The fermion closed loop and all ladder diagrams are included in the polarization operator and the Hartree-Fock single-particle Green's function are used for the fermion lines, see Fig. 2226 The Hartree-Fock random phase approximation is equivalent to a time dependent HartreeFock approximation. Since the time dependent Hartree-Fock approximation is a conserving 
approximation,27 the longitudinal f-sum-rule (9) is in general satisfied and serves as a test of the consistency of our numerical procedure. By using the Matsubara technique 28 (introducing the complex frequency $i \hbar \omega_{n}=i 2 n \pi k_{b} T$ where $n$ is an integral number, $k_{b}$ is the Boltzmann constant and $T$ is the temperature) to find the two-particle Green's function at finite temperatures, the matrix equation

$$
\begin{aligned}
& \Pi_{\alpha \beta ; \gamma \delta}\left(i \omega_{n}\right)=\Pi_{\gamma \delta}^{0}\left(i \omega_{n}\right) \delta_{\gamma, \beta} \delta_{\alpha, \delta}+ \\
& \Pi_{\gamma \delta}^{0}\left(i \omega_{n}\right) \sum_{\gamma^{\prime} \delta^{\prime}}\left(V_{\gamma^{\prime} \delta ; \gamma \delta^{\prime}}-V_{\delta \gamma^{\prime} ; \gamma \delta^{\prime}}\right) \Pi_{\alpha \beta ; \gamma^{\prime} \delta^{\prime}}\left(i \omega_{n}\right),
\end{aligned}
$$

is obtained where $\Pi_{\gamma \delta}^{0}\left(i \omega_{n}\right)$ is the unperturbed two-particle Green's function. It is given by the single-particle Hartree-Fock Green's functions

$$
\begin{aligned}
\Pi_{\gamma \delta}^{0}\left(i \omega_{n}\right) & =\frac{1}{\beta \hbar} \sum_{i \omega_{n^{\prime}}} G_{\gamma}\left(i \omega_{n}+i \omega_{n^{\prime}}\right) G_{\delta}\left(i \omega_{n^{\prime}}\right) \\
& =\frac{f_{\delta}-f_{\gamma}}{i \hbar \omega_{n}-\left(\epsilon_{\gamma}-\epsilon_{\delta}\right)}
\end{aligned}
$$

and the Coulomb matrix element is

$$
V_{\alpha \gamma ; \delta \beta}=\frac{e^{2}}{\kappa} \int d \boldsymbol{r} \int d \boldsymbol{r}^{\prime} \frac{\psi_{\alpha}^{*}(\boldsymbol{r}) \psi_{\beta}(\boldsymbol{r}) \psi_{\gamma}^{*}\left(\boldsymbol{r}^{\prime}\right) \psi_{\delta}\left(\boldsymbol{r}^{\prime}\right)}{\left|\boldsymbol{r}-\boldsymbol{r}^{\prime}\right|}
$$

The negative term in the summation (15) represents the vertex corrections. In the Hartree random phase approximation, this term is neglected. We now introduce the induced "density" matrices $K_{c d}^{ \pm}(\boldsymbol{q}, \omega)$

$$
K_{c d}^{ \pm}(\boldsymbol{q}, \omega)=\sum_{a b}\left\langle a\left|e^{i \boldsymbol{q} \cdot \boldsymbol{r}}\right| b\right\rangle \Pi_{a b ; c d}^{ \pm}(\omega)
$$

in order that the correlation functions may be expressed as

$$
\chi^{ \pm}(\boldsymbol{q}, \omega)=\sum_{c d} K_{c d}^{ \pm}(\boldsymbol{q}, \omega)\left\langle c\left|e^{-i \boldsymbol{q} \cdot \boldsymbol{r}}\right| d\right\rangle
$$

The charge-density induced "density" matrix satisfies the equation

$$
\begin{aligned}
& K_{a b}^{+}(\boldsymbol{q}, \omega)=\frac{f_{b}-f_{a}}{\hbar \omega-\left(\epsilon_{a}-\epsilon_{b}\right)}\left[2\left\langle a\left|e^{-i \boldsymbol{q} \boldsymbol{r}}\right| b\right\rangle^{*}+\right. \\
& \left.\sum_{c d}\left(2 V_{c b ; a d}-V_{b c ; a d}\right) K_{c d}^{+}(\boldsymbol{q}, \omega)\right],
\end{aligned}
$$


and similarly for the induced spin-density matrix

$$
\begin{aligned}
& K_{a b}^{-}(\boldsymbol{q}, \omega)=\frac{f_{b}-f_{a}}{\hbar \omega-\left(\epsilon_{a}-\epsilon_{b}\right)}\left[2\left\langle a\left|e^{-i \boldsymbol{q} \boldsymbol{r}}\right| b\right\rangle^{*}-\right. \\
& \left.\sum_{c d} V_{b c ; a d} K_{c d}^{-}(\boldsymbol{q}, \omega)\right] .
\end{aligned}
$$

If vertex corrections are neglected the spin-density excitation spectra are simply given by single-particle Hartree-Fock energies. In HF-RPA spin-density excitations are shifted from the single-particle Hartree-Fock energies due to the exchange interaction. As a result collective spin-density excitations may appear in the spectra. The shift from the single-particle Hartree-Fock energies is a measure of the strength of the exchange interaction in the system. For long wavelength charge-density excitations the direct Coulomb interaction dominates giving rise to plasmons, so that the exchange interaction is not so important here. It will however give a different coupling of the single-particle excitations and collective excitation (Landau damping). The eigenequations (20) and (21) have to be solved numerically. See Appendix B for a more detailed description of the numerical approach.

\section{INTERSUBBAND EXCITATIONS}

In the conventional backscattering geometry for Raman experiments 13 the wavelength of the incident and scattered light are much longer than the width of the wire. The dipole approximation is therefore valid for intersubband excitations. Far infrared absorption measurements in the transverse direction provide the same information as polarized Raman scattering, which in the case of parabolic confinement is simply that the generalized Kohn's theorem is satisfied giving a single peak in the spectra at $\hbar \omega_{0}$. Intersubband spin-density excitations may supply interesting additional spectroscopic information. In the noninteracting case and within the dipole approximation the spectral function is

$$
\begin{aligned}
S^{0}\left(q_{y}, \omega\right)= & {\left[\delta\left(\hbar \omega-\hbar \omega_{0}\right)-\delta\left(\hbar \omega+\hbar \omega_{0}\right)\right] \times } \\
& \pi\left(\frac{\hbar^{2} q_{y}^{2}}{2 m^{*}}\right) /\left(\hbar \omega_{0}\right)
\end{aligned}
$$


and the static susceptibility is

$$
R^{0}\left(q_{y}\right)=\left(\frac{\hbar^{2} q_{y}^{2}}{m^{*}}\right) /\left(\hbar \omega_{0}\right)^{2}
$$

for both the spin-density correlation function and charge-density correlation function. The charge-density correlation function does not depend on the Coulomb interaction according to the generalized Kohn's theorem in the dipole limit. Therefore $S\left(q_{y}, \omega\right)$ and $R\left(q_{y}\right)\left(q_{y} \rightarrow 0\right)$ are invariant with respect to the electronic density for charge-density excitations.

In the calculation of the Hartree-Fock ground state, the functional basis has been chosen large enough in order that further expansion of it or further iteration of the Hartree-Fock equations does not result in visual change to the single-particle energy spectra or the electron density of the ground state. To attain sufficient accuracy in the calculation of the chargedensity excitations and the spin-density excitations the size of the functional basis of the excited states has been chosen such that further refinement results in the change of the location of the excitation peaks smaller than a typical linewidth in experiments $\hbar \eta \approx 0.1 \mathrm{meV}$. For the calculations we employ the usual GaAs parameters, $m^{*}=0.067 m_{0}$, where $m_{0}$ is the electron mass, and the dielectric constant $\kappa=12.4$. The calculations have been performed for $T=1.0 \mathrm{~K}$.

We have calculated the intersubband spin-density excitations as a function of the electron density for two different confining potentials; $\hbar \omega_{0}=11.37 \mathrm{meV}\left(l_{0}=100 \AA\right)$ and $\hbar \omega_{0}=7.90 \mathrm{meV}$ $\left(l_{0}=120 \AA\right)$. The length of the wire in both calculations is $L_{x}=2400 \AA$. In all our results, the generalized Kohn's theorem is satisfied with a high degree of accuracy for charge-density excitations, i.e. there is only a single peak in the spectra at $\hbar \omega_{0}$ having all the spectral intensity satisfying the f-sum rule (9) as given in (22). The generalized Kohn's theorem is also satisfied within the Hartree random phase approximation. Therefore a Hartree RPA and a Hartree-Fock RPA calculation will give the same result for the long wavelength intersubband charge-density excitations for a parabolic confined wire.

Numerical results for the spin-density excitations as a function of the electronic density are shown in Fig. 3 where $\hbar \omega_{0}=11.37 \mathrm{meV}$ and Fig. 1 where $\hbar \omega_{0}=7.90 \mathrm{meV}$. The upper 
panel of Figs. 3 and 4 shows the static spin-density susceptibility and the center graph the excitation energy of the dominant peak in the spectra (SDE). The lowest subfigure shows the difference in energy between states at the zone center and the chemical potential for different subbands $\left(\epsilon_{n, k=0}-\mu\right)$. States below the horizontal line are occupied. The dominant peaks in the spectra have intensities that are typically one order of magnitude larger than the weaker peaks in the spectra in the density range shown. The intersubband SDE energy is red-shifted with respect to the single-particle Hartree-Fock energies. The excitation energy oscillates with the subband filling and has local minima where a new subbands starts to be filled. In this case the screening is large so that the self-consistent subband spacing is significantly smaller than the confinement energy $\hbar \omega_{0}$. In Fig. 5 the spin-density excitation with the largest oscillator strength has been calculated for densities common in present experiments on wires. In this calculation only, we use the local density approximation (LDA) for the exchange interaction with the parametrization 29

$$
V_{x}^{L D A}(y)=-2 \sqrt{\frac{2 n^{2 D}(y)}{\pi}} \frac{e^{2}}{\kappa} .
$$

For high density the oscillations of the spin-density excitations are weak. When the 2D electronic density is sufficiently low and we are close to a new subband starting to be filled, we see that the HF-RPA gives overdamped modes (imaginary excitation energy, see Appendix B) for the spin-density excitation, e.g. for $\hbar \omega_{0}=11.37 \mathrm{meV}\left(l_{0}=100 \AA\right)$ there is an overdamped region $n_{1 D}=(7.1-8.3) \times 10^{5} \mathrm{~cm}^{-1}$ and for $\hbar \omega_{0}=7.90 \mathrm{meV}\left(l_{0}=120 \AA\right)$ there are overdamped regions $n_{1 D}=(4.6-7.5,12.1-14.5) \times 10^{5} \mathrm{~cm}^{-1}$ for the densities shown. The presence of overdamped modes means that our Hartree Fock ground state is not stable in these density regimes. The oscillations in the excitation energy are followed by peaks in the static paramagnetic susceptibility and the static paramagnetic susceptibility is divergent in

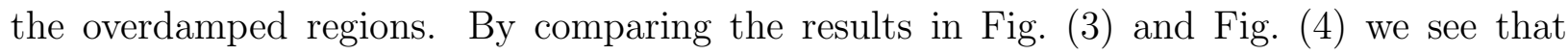
there are broader unstable regimes for $\hbar \omega_{0}=11.37 \mathrm{meV}\left(l_{0}=100 \AA\right)$ than for $\hbar \omega_{0}=7.90 \mathrm{meV}$ $\left(l_{0}=120 \AA\right)$ as should be expected since the electronic density is lower in the latter case and the validity of the HFA is not so good. Almost the same instability regions are found in the 
case of the LDA where correlation effects are neglected (24).

\section{INTRASUBBAND EXCITATIONS}

When the wave vector component along the wire is not zero, intrasubband excitations shows up in the spectra. We will first consider the case of three subbands occupied. For the case of two subbands a comparison with experiments has been briefly communicated in Ref. 30. Finally we study how the virtual transitions to upper nonoccupied subbands may alter the elementary excitation energies in the Tomonaga-Luttinger model, for only a single subband occupied.

\section{A. Low- and high-energy excitations: Numerical results}

The external parabolic potential was set to $11.37 \mathrm{meV}\left(l_{0}=100 \AA\right)$ resulting in a selfconsistent Hartree-Fock subband spacing between the lowest subbands of $6.6 \mathrm{meV}$ and between the next lowest subbands of $8.3 \mathrm{meV}$ at the zone center. Three subbands were occupied with densities $n_{0}=9.4 \times 10^{5} \mathrm{~cm}^{-1}, n_{1}=7.3 \times 10^{5} \mathrm{~cm}^{-1}$ and $n_{2}=3.3 \times 10^{4} \mathrm{~cm}^{-1}$. We take the wire to have a length of $L_{x}=1.0 \mu \mathrm{m}$.

For three subbands occupied it is generally expected目 6 that the SDE dispersions are linear in $q$, with sound velocities $v_{0}, v_{1}$ and $v_{2}$ for intrasubband excitations in the three lowest subbands. The Fermi velocities are given by the subband densities $v_{i}=\pi \hbar n_{i} / m^{*}(i=0,1,2)$. The CDE dispersion also has three branches. The in-phase mode is approximately 4

$$
\omega_{\rho}^{+}(q)=|q| \sqrt{2\left(v_{0}+v_{1}+v_{2}\right) V(q) / \hbar \pi},
$$

where $V(q)$ is the Fourier transform of the Coulomb potential $\left(e^{2} / \kappa\right)\left[\left(x-x^{\prime}\right)^{2}+l_{0}^{2}\right]^{-1 / 2}$. In addition there are two out-of-phase modes with linear dispersion.6

We note in general good qualitative agreement of our numerical results with the measured Raman spectra.9. CDE corresponding to one-dimensional intrasubband motion of the electron gas. The SDE, 
as can be seen in Fig. 6 follow a linear dispersion in the long wavelength limit. For three subbands occupied we see the three collective spin density modes with group velocities at $\tilde{v}_{0}, \tilde{v}_{1}$ and $\tilde{v}_{2}$. The Landau damping destroys the higher energy modes at large enough wavevectors. The Landau damping shows up in enhanced intensities of satellite single-particle peaks around the SDE, as shown in Fig. 6, since we considered a wire of a finite length $\left(L_{x}=1.0 \mu \mathrm{m}\right)$. For infinite wires one should expect a large number of SPE peaks merging into a broad band. We found the sound velocity $\tilde{v}_{0}$ larger than the corresponding Fermi velocity $\left(\tilde{v}_{0}=1.02 v_{0}\right)$, and the sound velocities $\tilde{v}_{1}$ and $\tilde{v}_{2}$ smaller than the corresponding Fermi velocities, $\left(\tilde{v}_{1}=0.98 v_{1}\right.$ and $\left.\tilde{v}_{2}=0.64 v_{2}\right)$. This is caused by the exchange interaction within each subband and intersubband coupling giving rise to renormalized sound velocities.2230 The other factor comes from intersubband virtual transitions due to exchange interaction leading to exchange screening of SDE. These low-energy features within the HF-RPA must be considered as approximate results.

The $q$ dependence of the intrasubband plasmon energy $(\mathrm{CDE}+)$ can be fitted by $q[-$ $\ln q]^{-1 / 2}$, as shown in the inset in Fig. [7. That is what is expected for one-dimensional wires. 422 For three subbands occupied there must also be two CDEs having linear dispersion which may also be seen in Fig. 7. The intensities of these excitations are however weak and maybe difficult to observe experimentally. The plasmon decays at higher wave-vectors due to the Landau damping, as seen in Fig. 7 .

The calculations performed in Refs. 6 9 within the Hartree random phase approximation have been demonstrated to agree very well with experimental results for the charge-density excitations. In order to see the effect of the vertex correction on the intrasubband chargedensity excitations we have also performed a calculation with the same sample parameters as above, but omitting the exchange interaction (not shown). Indeed, we found that the vertex corrections have negligible effect on the in-phase intrasubband plasmon energy. It agrees within the numerical accuracy $(1 \%)$. For the two out-of-phase modes the Hartree calculation gives a Fermi velocity $15 \%$ smaller for the highest energy out-of-phase mode and almost identical for the lowest out-of-phase mode as compared to the HF-RPA calculation. 
The HF-RPA generally gives about twice as large relative intensity of the out-of-phase modes relative to the in-phase intensity as compared to the Hartree random phase approximation.

It should be noted that according to our analysis of the wave-vector dependence of intrasubband SDE the frequency of the lowest energy SDE decreases at large $q \leq k_{2}$, and at some value of $q$ close to $2 k_{2}$ it goes to zero. That means an intrinsic instability of the system with respect to formation of spin density waves (SDW) below some critical temperature. This is the well-known Peierls instability of one-dimensional systems. Overhauser 13 showed that the HF paramagnetic state in 3D systems is always unstable with respect to formation of a static spin-density wave having a wave vector $q \approx 2 k_{F}$, and MacDonald 25 found that the same was expected in 2D systems. Therefore the Hartree-Fock spin susceptibility of the paramagnetic state has a singularity near $q=2 k_{F}$. The instability in the quasi $1 \mathrm{D}$ wire emerges from our mean-field analysis, and in the framework of this approach one should take into account SDW long-range order in the ground state. However, exact results making use of the Luttinger model predict no SDW long-range order, but predict a slowly decaying Wigner crystal of short range order at $4 k_{F}$.22

\section{B. Screening in the Tomonaga-Luttinger model}

In the Tomonaga-Luttinger model, the Coulomb interaction is restricted to processes within the lowest occupied subband and the kinetic energy is approximated by a linear dispersion.20 In this way the elementary low-energy excitations may be found exactly by using a bosonization technique. We will now study how the excitation energies for the Tomonaga-Luttinger model may change when the coupling to the higher subbands is taken into account. The virtual transitions to the upper subbands will give rise to direct and exchange screening. A second order perturbation argument shows that contributions from intersubband transitions will be inversely proportional to the subband spacing. We will now give some more quantitative results derived from an effective low-energy Hamiltonian where

the virtual high-energy intersubband excitations are treated within the HF-RPA. In quantum 
wires used in Raman measurements2 2 , coupling to higher subbands is always present and may not be small. Since this coupling may renormalize the excitation energies appearing in the experimental system it is an important physical parameter. The renormalization factors may be measured directly in an experiment if one is able to control the subband spacing (i.e. the curvature of the parabolic confinement) while keeping the 1D electronic density of the lowest subband constant.

For the low-energy excitations the interaction may be approximated by

$$
\begin{aligned}
\hat{V} & =\sum_{i j, q k p, s \sigma}\left[H^{i j}(k, p) \hat{c}_{i \sigma}^{\dagger}(p-q / 2) \hat{c}_{j \sigma}(p+q / 2) \hat{c}_{0 s}^{\dagger}(k+q / 2) \hat{c}_{0 s}(k-q / 2)\right. \\
& \left.-F^{i j}(k, p) \hat{c}_{i s}^{\dagger}(k+q / 2) \hat{c}_{j \sigma}(p+q / 2) \hat{c}_{0 \sigma}^{\dagger}(p-q / 2) \hat{c}_{0 s}(k-q / 2)\right],
\end{aligned}
$$

where the first term represents the Hartree interaction

$$
H^{i j}(k, p)=V^{i 0 ; 0 j}(p, k, k, p)
$$

and the second the Fock interaction

$$
F^{i j}(k, p)=V^{0 i ; 0 j}(k, p, k, p)
$$

between the lowest subband and the higher subbands. The matrix elements are evaluated within the Hartree-Fock basis, $V^{i j ; m n}(k p ; q r)=\int d \boldsymbol{r} \int d \boldsymbol{r}^{\prime} \psi_{i, k}^{*}(\boldsymbol{r}) \psi_{n, r}(\boldsymbol{r}) \psi_{j, p}^{*}\left(\boldsymbol{r}^{\prime}\right) \psi_{m, q}\left(\boldsymbol{r}^{\prime}\right) / \mid \boldsymbol{r}-$ $\boldsymbol{r}^{\prime} \mid$. By adding this interaction part to the TLM Hamiltonian the virtual transitions to the upper subbands are included. Following the standard bosonization procedure 20.28 we now introduce the boson operators for charge-density excitations $\hat{b}^{+}$and spin-density excitations $\hat{b}^{-}$in the lowest subband $(q>0)$,

$$
\sum_{k>0}\left(\hat{c}_{0 \uparrow}^{\dagger}(k+q / 2) \hat{c}_{0 \uparrow}(k-q / 2) \pm \hat{c}_{0 \downarrow}^{\dagger}(k+q / 2) \hat{c}_{0 \downarrow}(k-q / 2)\right)=\hat{b}^{ \pm}(q) \sqrt{\frac{q L_{x}}{\pi}}
$$

and

$$
\sum_{k<0}\left(\hat{c}_{0 \uparrow}^{\dagger}(k-q / 2) \hat{c}_{0 \uparrow}(k+q / 2) \pm \hat{c}_{0 \downarrow}^{\dagger}(k-q / 2) \hat{c}_{0 \downarrow}(k+q / 2)\right)=\hat{b}^{ \pm}(-q) \sqrt{\frac{q L_{x}}{\pi}} .
$$

The spin and charge parts of the Hamiltonian decouple so that the interaction Hamiltonian may be written as $\hat{V}=\hat{V}^{+}+\hat{V}^{-}$, 


$$
\hat{V}^{ \pm}=\sum_{i j, k q} \hat{\psi}_{i j}^{ \pm}(k, q)\left(X_{1}^{ \pm i j}(k, q) \hat{b}(q)+X_{2}^{ \pm i j}(k, q) \hat{b}^{\dagger}(-q)\right)
$$

where we have introduced the intersubband charge-density and spin-density operators

$$
\begin{aligned}
\hat{\psi}_{i j}^{ \pm}(k, q)= & \hat{c}_{i \uparrow}(k-q / 2) \hat{c}_{j \uparrow}(k+q / 2) \pm \\
& \hat{c}_{i \downarrow}(k-q / 2) \hat{c}_{j \downarrow}(k+q / 2),
\end{aligned}
$$

the charge-density matrix element $(n=1,2)$

$$
X_{n}^{+i j}(k, q)=\sqrt{\left|\frac{q L_{x}}{\pi}\right|}\left(H^{i j}\left(k_{F}, k\right)-\frac{1}{2} F_{n}^{i j}(k, q)\right),
$$

and the spin-density matrix elements

$$
X_{n}^{-i j}(k, q)=\sqrt{\left|\frac{q L_{x}}{\pi}\right|}\left(-\frac{1}{2} F_{n}^{i j}(k, q)\right) .
$$

Here $F_{n}^{i j}(k, q)$ are combinations of the Fock matrix at $k_{F}$ and $-k_{F}$

$$
F_{1}^{i j}(k, q)=F^{i j}\left(k_{F}, k\right) \theta(q)+F^{i j}\left(-k_{F}, k\right) \theta(-q)
$$

and

$$
F_{2}^{i j}(k, q)=F^{i j}\left(k_{F}, k\right) \theta(-q)+F^{i j}\left(-k_{F}, k\right) \theta(q) .
$$

There are also spin-flip terms in the interaction Hamiltonian similar to the spin-spin term in (31), but the spin triplet is degenerate so we can limit the discussion to only one of the modes. The TLM may be solved by performing the Bogoliubov transformation 20,28

$$
\begin{gathered}
\hat{b}^{ \pm}(q)+\hat{b}^{ \pm \dagger}(-q)=\frac{\hbar \omega(q)}{E^{ \pm}(q)}\left(\hat{\beta}^{ \pm}(q)+\hat{\beta}^{ \pm \dagger}(-q)\right) \\
\hat{b}^{ \pm}(q)-\hat{b}^{ \pm \dagger}(-q)=\frac{\hbar \omega(q)}{E^{ \pm}(q)}\left(\hat{\beta}^{ \pm \dagger}(q)-\hat{\beta}^{ \pm}(-q)\right),
\end{gathered}
$$

where $\omega(q)=v_{F}|q|$ is the unperturbed excitation frequency and $E^{ \pm}(q)$ is the excitation energy of the charge-density and spin-density excitations given by the one-band Luttinger model. In order to find the elementary intrasubband excitations in the system we define the time-ordered boson Green's functions 


$$
D^{ \pm}(q, \omega)=-\frac{i}{\hbar} \int_{-\infty}^{\infty} e^{i \omega t}\left\langle\left|T \hat{\beta}^{ \pm}(q, t) \hat{\beta}^{ \pm \dagger}(q, 0)\right|\right\rangle
$$

and

$$
D_{A}^{ \pm}(q, \omega)=-\frac{i}{\hbar} \int_{-\infty}^{\infty} e^{i \omega t}\left\langle\left|T \hat{\beta}^{ \pm \dagger}(-q, t) \hat{\beta}^{ \pm \dagger}(q, 0)\right|\right\rangle
$$

where the ordinary unperturbed (coupling to the higher subbands is neglected) Green's functions is $D_{0}^{ \pm}(q, \omega)=1 /\left(\hbar \omega-E^{ \pm}(q)\right)$ and the anomalous unperturbed Green's function is $D_{A, 0}^{ \pm}(q, \omega)=0$. The interaction Hamiltonian (31) may be represented in the same form as (31)

$$
\hat{V}^{ \pm}=\sum_{q}\left[\hat{f}_{1}^{ \pm 1}(q) \hat{\beta}^{ \pm}(q)+\hat{f}_{2}^{ \pm 2}(q) \hat{\beta}^{ \pm \dagger}(-q)\right]
$$

where $\hat{f}_{1}^{ \pm 1}(q)$ and $\hat{f}_{2}^{ \pm 2}(q)$ are given by the intersubband operators (32), the matrix elements (33) and (34), and the transformation (37). To lowest order in the intersubband coupling the Dyson's equations are

$$
\begin{aligned}
D^{ \pm}(\omega)= & D_{0}^{ \pm}(\omega)+ \\
& D_{0}^{ \pm}(\omega)\left[P_{12}^{ \pm} D^{ \pm}(\omega)+P_{11}^{ \pm} D_{A}^{ \pm}(\omega)\right]
\end{aligned}
$$

and

$$
D_{A}^{ \pm}(\omega)=0+D_{0}^{ \pm}(-\omega)\left[P_{12} D_{A}^{ \pm}(\omega)+P_{11} D^{ \pm}(\omega)\right]
$$

where the polarizations $P_{\alpha \beta}^{ \pm}(q, \omega)(\alpha, \beta=1,2)$ are defined as

$$
P_{\alpha \beta}^{ \pm}(q, \omega)=-\frac{i}{\hbar} \int_{-\infty}^{\infty} d t e^{i \omega t}\left\langle\left|T \hat{f}_{\alpha}^{ \pm}(-q, t) \hat{f}_{\beta}^{ \pm}(q, 0)\right|\right\rangle
$$

and may be expressed in terms of the time-ordered two-particle operator $\Pi^{T \pm}$ given by similar equations as the retarded two-particle Green's functions $\Pi^{ \pm}$in (13). Intersubband excitations are nearly vertical and the intersubband excitation energies are much larger than the low-energy intrasubband excitation energy, hence we approximate the polarizations (43) by it's limit when $(q, \omega) \rightarrow(0,0)$. The poles of the Green's function (38) defines the renormalized intrasubband excitation energies 


$$
\tilde{E}^{ \pm}(q)^{2}=E^{ \pm}(q)^{2}+2 P_{12} E^{ \pm}(q)+\left(P_{12}^{2}-P_{11}^{2}\right)
$$

The virtual transitions to the higher subbands are included by the polarization operator (43) that describes the coupling between the lowest occupied subband and all the higher subbands. By carrying out this calculation we find that the shift of the excitation energies may be represented by the dimensionless parameters $(n=1,2)$

$$
h_{n}^{ \pm}=-\frac{2 L_{x}}{v_{F} \hbar \pi} \sum_{i j l m, k p} W_{n}^{ \pm i j}(k) W_{n}^{ \pm l m}(p) \Pi_{i j l m}^{T \pm}(k, p)
$$

where $W_{1}^{ \pm i j}(k)$ and $W_{2}^{ \pm i j}(k)$ are given by

$$
\begin{aligned}
W_{1}^{+i j}(k) & =H^{i j}\left(k_{F}, k\right) \\
& -\frac{1}{4}\left(F^{i j}\left(k_{F}, k\right)+F^{i j}\left(-k_{F}, k\right)\right), \\
W_{1}^{-i j}(k) & =-\frac{1}{4}\left(F^{i j}\left(k_{F}, k\right)+F^{i j}\left(-k_{F}, k\right)\right),
\end{aligned}
$$

and

$$
W_{2}^{ \pm i j}(k)=-\frac{1}{4}\left(F^{i j}\left(k_{F}, k\right)-F^{i j}\left(-k_{F}, k\right)\right)
$$

The intrasubband excitation energies are

$$
\left(\frac{\tilde{E}^{ \pm}(q)}{E^{ \pm}(q)}\right)^{2}=\left(1-\left(\frac{\hbar \omega(q)}{E^{ \pm}(q)}\right)^{2} \tilde{h}_{1}^{ \pm}\right)\left(1-\tilde{h}_{2}^{ \pm}\right)
$$

where $h_{1}^{ \pm}$and $h_{2}^{ \pm}$represent the contribution caused by screening due to the virtual excitations to the nonoccupied upper subbands. Stability of the system with respect to compression requires the screening factors not to be too strong. If not, then the perturbation approach is not valid since we see from (49) that too strong screening factors lead to imaginary intrasubband excitation energies. For charge-density excitations the plasmon energy has the low-energy behavior $q[-\ln q]^{1 / 2}$ so that the renormalization factor $h_{1}^{+}$is unimportant. The screening factors $h_{2}^{ \pm}$are given by differences of the Fock matrix elements with respect to $k_{F}$ and $-k_{F}(48)$ and are therefore expected to be small. 
We have evaluated the intersubband screening factors $h_{1}^{ \pm}$and $h_{2}^{ \pm}$for a wire of density $n_{1 D}=2.0 \times 10^{5} \mathrm{~cm}^{-1}$ and for subband separation starting at $2 \mathrm{meV}$ to $46 \mathrm{meV}$, as shown in Fig. (8). The screening factor $h_{2}^{ \pm}$appears in the calculation orders of magnitude smaller than $h_{1}^{ \pm}$and may be neglected, as already pointed out above. Even at an external potential separation of $2 \mathrm{meV}$ they are smaller than $1 \%$. Since the effect of the screening factor $h_{1}^{+}$ is unimportant, we find that the plasmon energy is rather insensitive to the coupling to higher subbands. Screening has a larger effect on spin-density excitations. The effect on the SDE sound velocity is about $5 \%$ down to a subband spacing of $4 \mathrm{meV}$. For smaller subband spacings, the exchange screening factor $h_{1}^{-}$appears to decrease, but this is due to the fact that the HFA is not valid in this small subband separation regime, because the spin-density excitations are overdamped. The screening parameters $h_{1}^{ \pm}$decrease inversely

proportional to the subband spacing as expected, but the factors $h_{2}^{ \pm}$decrease faster than inversely proportional to the square of the subband spacing. The screening parameters are not very sensitive to the $1 \mathrm{D}$ electronic density. A similar calculation for $n_{1 D}=3 \times 10^{5} \mathrm{~cm}^{-1}$ (not shown) shows that the factor $h_{1}^{+}$decreases by less than $10 \%$ at $\hbar \omega_{0}=5 \mathrm{meV}$ as compared to the results in Fig. (8). Similarly the factor $h_{1}^{-}$decreases by less than $20 \%$ at $\hbar \omega_{0}=5 \mathrm{meV}$.

\section{CONCLUSIONS}

The importance of the vertex corrections (exchange interaction) that give rise to collective spin-density excitations in quantum wires is demonstrated. For charge-density excitations vertex corrections are less important. The calculated spin-density excitation spectra and charge-density excitation spectra seem to agree well with experiments. The dipole intersubband spin-density excitation energy oscillates with the number of occupied subbands accompanied by peaks in the static spin-density susceptibility. For a single subband occupied screening by virtual transitions to the upper subband renormalizes the spin-density energy by the same order of magnitude as the correction due to backscattering mixing rightand left-moving modes. 


\section{ACKNOWLEDGMENTS}

A. B. would like to thank A. Sudbø and L. J. Sham for stimulating discussions and M. Willander for a great hospitality at the Linkøping University where parts of this work was carried out. This work has been supported in part by a NorFa grant, the Icelandic Science Foundation, the University of Iceland Research Fund, the German Science Foundation DFG, and the Research Council of Norway (Program for Supercomputing) through a grant of computing time.

\section{APPENDIX A: SINGULAR CUT-OFF}

In an infinite wire the ground state energy of an interacting electron gas without background charges is divergent, which requires a cut-off introduced by background charges for the low-wave vector interaction $q$ similar to the 3D situation. Consider the Hamilton operator in the basis of the noninteracting states

$$
\psi_{n k}(x, y)=\frac{1}{\sqrt{L_{x}}} e^{i k x} \phi_{n}(y)
$$

The Coulomb interaction in the noninteracting basis is

$$
\hat{H}_{i n t}=\frac{1}{2} \sum_{i m l j} \sum_{k k^{\prime} q} \sum_{\sigma \sigma^{\prime}} V_{0}^{i m ; l j}(q) \hat{c}_{i \sigma}^{\dagger}(k-q) \hat{c}_{m \sigma^{\prime}}^{\dagger}\left(k^{\prime}+q\right) \hat{c}_{l \sigma^{\prime}}\left(k^{\prime}\right) \hat{c}_{j \sigma}(k)
$$

where $\hat{c}_{i \sigma}(k)$ destroys an electron in a state with transverse quantum number $i$, spin $\sigma$ and longitudinal wave-vector $k$ and the Coulomb matrix elements are

$$
\begin{aligned}
& V_{0}^{i m ; l j}(q)= \\
& \frac{e^{2}}{\kappa} \int d \boldsymbol{r} \int d \boldsymbol{r}^{\prime} \frac{1}{L_{x}^{2}} \frac{e^{i q\left(x-x^{\prime}\right)} \phi_{i}(y) \phi_{j}(y) \phi_{m}\left(y^{\prime}\right) \phi_{l}\left(y^{\prime}\right)}{\left|\boldsymbol{r}-\boldsymbol{r}^{\prime}\right|}
\end{aligned}
$$

which in the limit of long wires can be written as

$$
\begin{aligned}
& V_{0}^{i m ; l j}(q)=\frac{2 e^{2}}{\kappa L_{x}} \times \\
& \int d y \int d y^{\prime} K_{0}\left(\left|q\left(y-y^{\prime}\right)\right|\right) \phi_{i}(y) \phi_{j}(y) \phi_{m}\left(y^{\prime}\right) \phi_{l}\left(y^{\prime}\right) .
\end{aligned}
$$


For small arguments the Bessel function is asymptotically $K_{0}(x) \sim-\ln x$. For small wave vectors $q$ we may therefore take away the divergent part in the matrix element

$$
\begin{aligned}
& V^{i m ; l j}(q)=-\frac{2 e^{2}}{\kappa L_{x}} \ln q l_{0} \delta_{i, j} \delta_{m, l}-\frac{2 e^{2}}{\kappa L_{x}} \times \\
& \int d y \int d y^{\prime} \ln \left|\frac{y-y^{\prime}}{l_{0}}\right| \phi_{i}(y) \phi_{j}(y) \phi_{m}\left(y^{\prime}\right) \phi_{l}\left(y^{\prime}\right) .
\end{aligned}
$$

For a finite system we may therefore write

$$
V^{i m ; l j}(q)=\delta_{q, 0} \delta_{i, j} \delta_{m, l} C+\tilde{V}^{i m ; l j}(q)
$$

where $C$ is a large constant. This first term in (A6) gives a part $C\left(\hat{N}^{2}-\hat{N}\right)$ to the Hamiltonian where $\hat{N}$ is the number operator of particles. We consider states where the total number of particles is a good quantum number so that this term is cancelled by a similar term from the background charges. The renormalized matrix element for finite wave vector $q$ is given by (A4) and for $q=0$

$$
\begin{aligned}
& \tilde{V}^{i m ; l j}(q=0)=-\frac{2 e^{2}}{\kappa L_{x}} \times \\
& \int d y \int d y^{\prime} \ln \left|\frac{y-y^{\prime}}{L}\right| \phi_{i}(y) \phi_{j}(y) \phi_{m}\left(y^{\prime}\right) \phi_{l}\left(y^{\prime}\right) .
\end{aligned}
$$

In the Hartree approximation this is the same as the long wire limit in Refs. [ 33, 34]. For an easier numerical evaluation by using the integral form of the Bessel function

$$
K_{0}(x)=\int_{-\infty}^{\infty} d t \frac{e^{i x t}}{2 \sqrt{t^{2}+1}}
$$

the integral (A4) may be written as

$$
\begin{aligned}
& V_{0}^{i m ; l j}(q)=\frac{e^{2}}{\kappa L_{x}} \times \\
& \int_{-\infty}^{\infty} d u \frac{1}{\sqrt{u^{2}+q^{2} l_{0}^{2}}} I_{i, j}(u) I_{m, l}(-u)
\end{aligned}
$$

where the function $I_{i, j}(u)$ is the Fourier transform of the product of a pair of transverse wave-functions

$$
I_{l, l^{\prime}}(u)=\int_{-\infty}^{\infty} d v \phi_{l}(v) \phi_{l^{\prime}}(v) e^{i u v t}
$$


and $u$ and $v$ are dimensionless variables. The function (A10) may be written as a linear combination of Laguerre functions, so only a one-dimensional integral has to be evaluated numerically in (A9).

\section{APPENDIX B: DIAGONALIZATION OF EXCITED STATES}

The eigenequations (20) and (21) are not well suited for a numerical diagonalization since they are in general not symmetric. In the case of the time dependent Hartree approximation this problem is easily solved due to the local nature of the approximation. 55 The nonlocal character of the Fock term causes some problems. However the eigenequations may be transformed to a symmetric form as will be described since this may be of interest for other calculations based on the Hartree-Fock random phase approximation. This appendix therefore covers some of the more technical aspects of the calculations.

By decoupling (20) or (21) into symmetric and antisymmetric combinations the eigenvalue equation may be rewritten. We define the new variables

$$
\begin{gathered}
y_{a b}^{S, \pm}(\boldsymbol{q}, \omega)=\frac{K_{a b}^{ \pm}(\boldsymbol{q}, \omega)+K_{b a}^{ \pm}(\boldsymbol{q}, \omega)}{\sqrt{\left(\epsilon_{a}-\epsilon_{b}\right)\left(f_{b}-f_{a}\right)}} \\
y_{a b}^{A, \pm}(\boldsymbol{q}, \omega)=\left(K_{a b}^{ \pm}(\boldsymbol{q}, \omega)-K_{a b}^{ \pm}(\boldsymbol{q}, \omega)\right) \sqrt{\frac{\epsilon_{a}-\epsilon_{b}}{f_{b}-f_{a}}}
\end{gathered}
$$

and

$$
\begin{aligned}
b_{a b}^{S}(\boldsymbol{q})= & 2 \sqrt{\left(f_{b}-f a\right)\left(\epsilon_{a}-\epsilon_{b}\right)}[ \\
& \left.\left\langle a\left|e^{-i \boldsymbol{q} \cdot \boldsymbol{r}}\right| b\right\rangle+\left\langle b\left|e^{-i \boldsymbol{q} \cdot \boldsymbol{r}}\right| a\right\rangle\right] \\
b_{a b}^{A}(\boldsymbol{q})= & 2 \sqrt{\frac{f_{b}-f a}{\epsilon_{a}-\epsilon_{b}}}\left(\left\langle a\left|e^{-i \boldsymbol{q} \cdot \boldsymbol{r}}\right| b\right\rangle-\left\langle b\left|e^{-i \boldsymbol{q} \cdot \boldsymbol{r}}\right| a\right\rangle\right)
\end{aligned}
$$

and restrict the summation to $\epsilon_{a}<\epsilon_{b}$ obtaining the matrix equations

$$
\begin{gathered}
-\omega \boldsymbol{y}^{A, \pm}(\boldsymbol{q}, \omega)=\mathcal{S}^{ \pm} \cdot \boldsymbol{y}^{S, \pm}(\boldsymbol{q}, \omega)+\boldsymbol{b}^{S, \pm}(\boldsymbol{q}) \\
-\omega \boldsymbol{y}^{S, \pm}(\boldsymbol{q}, \omega)=\mathcal{A}^{ \pm} \cdot \boldsymbol{y}^{A, \pm}(\boldsymbol{q}, \omega)+\boldsymbol{b}^{A, \pm}(\boldsymbol{q}),
\end{gathered}
$$


where the matrices (since all Coulomb matrix elements are real) $\mathcal{S}^{ \pm}$and $\mathcal{A}^{ \pm}$are symmetric and antisymmetric combinations of the Coulomb interaction elements defined by

$$
\begin{aligned}
\mathcal{S}_{a b, c d}^{+}= & \sqrt{\left(f_{b}-f_{a}\right)\left(f_{d}-f_{c}\right)\left(\epsilon_{a}-\epsilon_{b}\right)\left(\epsilon_{c}-\epsilon_{d}\right)}[ \\
& 2\left(V_{c b ; a d}+V_{c a ; b d}+V_{d b ; a c}+V_{d a ; b c}\right) \\
- & \left.\left(V_{b c ; a d}+V_{a c ; b d}+V_{b d ; a c}+V_{a d ; b c}\right)\right]
\end{aligned}
$$

and

$$
\begin{aligned}
\mathcal{A}_{a b, c d}^{+}= & \sqrt{\frac{\left(f_{b}-f_{a}\right)\left(f_{d}-f_{c}\right)}{\left(\epsilon_{a}-\epsilon_{b}\right)\left(\epsilon_{c}-\epsilon_{d}\right)}}[ \\
& 2\left(V_{c b ; a d}-V_{c a ; b d}-V_{d b ; a c}+V_{d a ; b c}\right) \\
- & \left.\left(V_{b c ; a d}-V_{a c ; b d}-V_{b d ; a c}+V_{a d ; b c}\right)\right]
\end{aligned}
$$

Similarly for the spin-density excitations the Hartree terms are absent. By now assuming that the matrix $\mathcal{A}$ is positive definite (this assumption has always been satisfied in our calculations), we can use a Cholesky decomposition $\mathcal{A}=\mathcal{L} \cdot \mathcal{L}^{T}$ where $\mathcal{L}$ is a real matrix and diagonalize the system. The excitation energies are given by the symmetric eigenvalue problem

$$
\mathcal{L}^{T} \cdot \mathcal{S} \cdot \mathcal{L} \cdot \mathcal{Z}=\mathcal{Z} \cdot \omega_{*}^{2}
$$

where the matrix $\mathcal{Z}$ contains the eigenvectors of the corresponding eigenvalues in the diagonal matrix $\omega_{*}^{2}$. If at least one eigenvalue in the matrix $\omega_{*}^{2}$ is negative, it means that we have overdamped modes. The Hartree-Fock ground state is then unstable.

The imaginary part of the charge-density and spin-density correlation functions can now be found to be

$$
\begin{aligned}
S(\boldsymbol{q}, \omega) & \equiv-\operatorname{Im}(\chi(\boldsymbol{q}, \omega)) \\
& = \pm \frac{\pi}{4} \sum_{x} \delta\left(\omega \mp \omega_{x}^{*}\right)\left(W_{x}^{S}(\boldsymbol{q}) \mp W_{x}^{A}(\boldsymbol{q})\right)^{2}
\end{aligned}
$$

where the weights $W_{x}^{S}(\boldsymbol{q})$ and $W_{x}^{S}(\boldsymbol{q})$ are defined as 


$$
\begin{gathered}
W_{x}^{A}(\boldsymbol{q})=\sum_{y z} b_{y}^{A}(\boldsymbol{q})\left(L_{y z}^{T}\right)^{-1} \mathcal{Z}_{z x} \sqrt{\omega_{x}^{*}} \\
W_{x}^{S}(\boldsymbol{q})=\sum_{y z} b_{y}^{S}(\boldsymbol{q}) L_{y z} \mathcal{Z}_{z x} \sqrt{1 / \omega_{x}^{*}}
\end{gathered}
$$

and the indices $x, y, z$ denotes electron-hole pair excitations. From (B7) it is seen that the charge-density and spin-density excitation intensities are positive as they should be. In general, the spectral function should have the symmetry $S(\boldsymbol{q}, \omega)=-S(-\boldsymbol{q},-\omega)$ since from (6) and (17) we see that $\chi(\boldsymbol{q}, \omega)=\chi^{*}(-\boldsymbol{q},-\omega)$. Here the weights have the symmetry $W_{x}^{S}(\boldsymbol{q})=W_{x}^{S}(-\boldsymbol{q})$ and $W_{x}^{A}(\boldsymbol{q})=-W_{x}^{A}(-\boldsymbol{q})$ so that $(\mathbb{B} 7)$ has the correct symmetry.

The real part of the correlation function

$$
R(\boldsymbol{q}, \omega)=-\operatorname{Re}(\chi(\boldsymbol{q}, \omega))
$$

may be found from the imaginary part by the Kramers-Kronig relation

$$
R(\boldsymbol{q}, \omega)=\frac{1}{\pi} \int_{-\infty}^{\infty} d \omega^{\prime} S\left(\boldsymbol{q}, \omega^{\prime}\right) \mathrm{P} \frac{1}{\omega^{\prime}-\omega}
$$

where $\mathrm{P}$ denotes the principal part. In the static case the susceptibility is therefore

$$
R(\boldsymbol{q})=\frac{1}{2} \sum_{x} \frac{\left(W_{x}^{S}(\boldsymbol{q})\right)^{2}+\left(W_{x}^{A}(\boldsymbol{q})\right)^{2}}{\omega_{x}^{*}} .
$$

The static susceptibility diverges if there are excitations with very low excitation energy and finite weight. 


\section{REFERENCES}

${ }^{1}$ T. Egeler et al., Phys. Rev. Lett. 65, 1804 (1990).

${ }^{2}$ A. R. Goñi et al., Phys. Rev. Lett. 67, 3298 (1991).

${ }^{3}$ A. Schmeller et al., Phys. Rev. B 49, 14778 (1994).

${ }^{4}$ Q. Li and S. D. Sarma, Phys. Rev. B 40, 5860 (1989).

${ }^{5}$ H. Yu and J. C. Hermanson, Phys. Rev. B 42, 1496 (1990).

${ }^{6}$ Q. Li and S. D. Sarma, Phys. Rev. B 43, 11768 (1991).

${ }^{7}$ L. Wendler and V. G. Grigoryan, Phys. Rev. B 49, 13607 (1994).

${ }^{8}$ F. A. Reboredo and C. R. Proetto, Phys. Rev. B 50, 15174 (1994).

${ }^{9}$ E. Hwang and S. D. Sarma, Phys. Rev. B 50, 17267 (1994).

${ }^{10}$ T. Ando, J. Phys. Soc. Jpn. 51, 3893 (1982).

${ }^{11}$ S. Katayama and T. Ando, J. Phys. Soc. Jpn 54, 1615 (1984).

${ }^{12}$ A. C. Tselis and J. J. Quinn, Phys. Rev. B 29, 3318 (1984).

${ }^{13}$ G. Eliasson, P. Hawrylak, and J. J. Quinn, Phys. Rev. B 35, 5569 (1987).

${ }^{14}$ W. Kohn, Phys. Rev. 123, 1242 (1961).

${ }^{15}$ D. C. Mattis and E. H. Lieb, J. Math. Phys. 6, 304 (1965).

${ }^{16}$ I. E. Dzyaloshinskii and A. I. Larkin, Zh. Eksp. Teor. Fiz 65, 411 (1973), [Sov. Phys. JETP 38, 202 (1974)].

${ }^{17}$ Q. P. Li, S. D. Sarma, and R. Joynt, Phys. Rev. B 45, 13713 (1992).

18 S. Tomonaga, Prog. Theor. Phys. (Kyoto) 5, 544 (1950).

19 J. M. Luttinger, J. Math. Phys. 4, 1154 (1963). 
${ }^{20}$ F. D. M. Haldane, J. Phys. C 14, 2585 (1981).

${ }^{21}$ A. Luther and V. J. Emery, Phys. Rev. Lett. 33, 589 (1974).

${ }^{22}$ H. J. Schulz, Phys. Rev. Lett. 71, 1864 (1993).

${ }^{23}$ D. C. Hamilton and A. L. McWhorter, in Light Scattering Spectra of Solids, edited by G. B. Wright (Springer, New York, 1969).

${ }^{24}$ C. Kallin and B. I. Halperin, Phys. Rev. B 30, 5655 (1984).

${ }^{25}$ A. H. MacDonald, J. Phys. C: Solid State Phys. 18, 1003 (1985).

${ }^{26}$ V. Gudmundsson and J. J. Palacios, Phys. Rev. B 52, 11266 (1995).

${ }^{27}$ G. Baym and L. P. Kadanoff, Phys. Rev. 124, 287 (1961).

${ }^{28}$ G. D. Mahan, Many-Particle Physics (Plenum Press, New York, 1990).

${ }^{29}$ B. Tanatar and D. M. Ceperley, Phys. Rev. B 39, 5005 (1989).

${ }^{30}$ A. Brataas, A. G. Mal'shukov, V. Gudmundsson, and K. A. Chao, J. Phys.: Condens. Matter 8, L325 (1996).

${ }^{31}$ A. W. Overhauser, Phys. Rev. 128, 1437 (1962).

32 B. Y.-K. Hu and S. D. Sarma, Phys. Rev. B 48, 5469 (1993).

${ }^{33}$ V. Gudmundsson, R. R. Gerhardts, R. Johnston, and L. Schweitzer, Z. Phys. B 70, 453 (1988).

${ }^{34}$ V. Gudmundsson et al., Phys. Rev. B 51, 17744 (1995).

${ }^{35}$ A. Brataas, V. Gudmundsson, A. G. Mal'shukov, and K. A. Chao, J. Phys.: Condens. Matter 8, 4797 (1996). 


\section{FIGURES}

FIG. 1. Feynman diagrams for the single-particle Green's function in the Hartree-Fock Approximation. The thick lines represent the Hartree-Fock single-particle propagator, the thin lines represent the noninteracting single-particle propagator and the dashed line is the electron-electron interaction.

FIG. 2. Feynman diagrams for the two-particle Green's function in the Hartree-Fock random phase approximation. The shaded boxes represent the four point vertex function, the thick lines represent the Hartree-Fock single-particle Green's function, and the dashed line is the electron-electron interaction.

FIG. 3. Intersubband spin-density excitations in the dipole approximation as a function of the electronic density. The upper panel shows the static susceptibility, the center graph the excitation energy of the dominant peak in the spectra, and the lowest subfigure the difference between the single-particle energy at the zone center and the chemical potential. The confinement energy is $\hbar \omega_{0}=11.37 \mathrm{meV}$ and the length of the wire $L_{x}=2400 \AA, T=1.0 \mathrm{~K}, m^{*}=0.067 m_{0}$ and $\kappa=12.4$.

FIG. 4. The same as in Fig. (3), except that the confinement energy is $\hbar \omega_{0}=7.90 \mathrm{meV}$.

FIG. 5. The energy of the strongest intersubband spin-density excitation in the dipole approximation as a function of the electron density for $\hbar \omega_{0}=7.90 \mathrm{meV}$ (upper panel), and $\hbar \omega_{0}=11.37$ meV (lower panel). Only here, the local density approximation has been used for the exchange interaction. Other parameters are as in Fig. 1 .

FIG. 6. Intrasubband spin-density excitations. Three subbands were occupied in the wire of length $L_{x}=1.0 \mu \mathrm{m}$ with densities $n_{0}=9.4 \times 10^{5} \mathrm{~cm}^{-1}, n_{1}=7.3 \times 10^{5} \mathrm{~cm}^{-1}$ and $n_{2}=3.3 \times 10^{5} \mathrm{~cm}^{-1}$. The external parabolic potential was $\hbar \omega_{0}=11.37 \mathrm{meV}\left(l_{0}=100 \AA\right)$. The inset shows the dispersion of the collective excitations. 
FIG. 7. Intrasubband charge-density excitations. Three subbands were occupied in the wire of length $L_{x}=1.0 \mu \mathrm{m}$ with densities $n_{0}=9.4 \times 10^{5} \mathrm{~cm}^{-1}, n_{1}=7.3 \times 10^{5} \mathrm{~cm}^{-1}$ and $n_{2}=3.3 \times 10^{5} \mathrm{~cm}^{-1}$. The external parabolic potential was $\hbar \omega_{0}=11.37 \mathrm{meV}\left(l_{0}=100 \AA\right)$. The inset shows the dispersion of the collective excitations.

FIG. 8. Screening parameters $\left(h_{1}^{+}, h_{2}^{+}, h_{1}^{-}\right.$and $\left.h_{2}^{-}\right)$as a function of the external parabolic confinement energy $\hbar \omega_{0}$. The dashed lines show the charge-density screening parameters $h_{1}^{+}$and $h_{2}^{+}$where $h_{1}^{+}$is larger than $h_{2}^{+}$. The straight lines show the spin-density screening parameters $h_{1}^{-}$ and $h_{2}^{-}$where $h_{1}^{-}$is larger than $h_{2}^{-}$. The electronic density of the wire is $n=2.0 \times 10^{5} \mathrm{~cm}^{-1}$. 


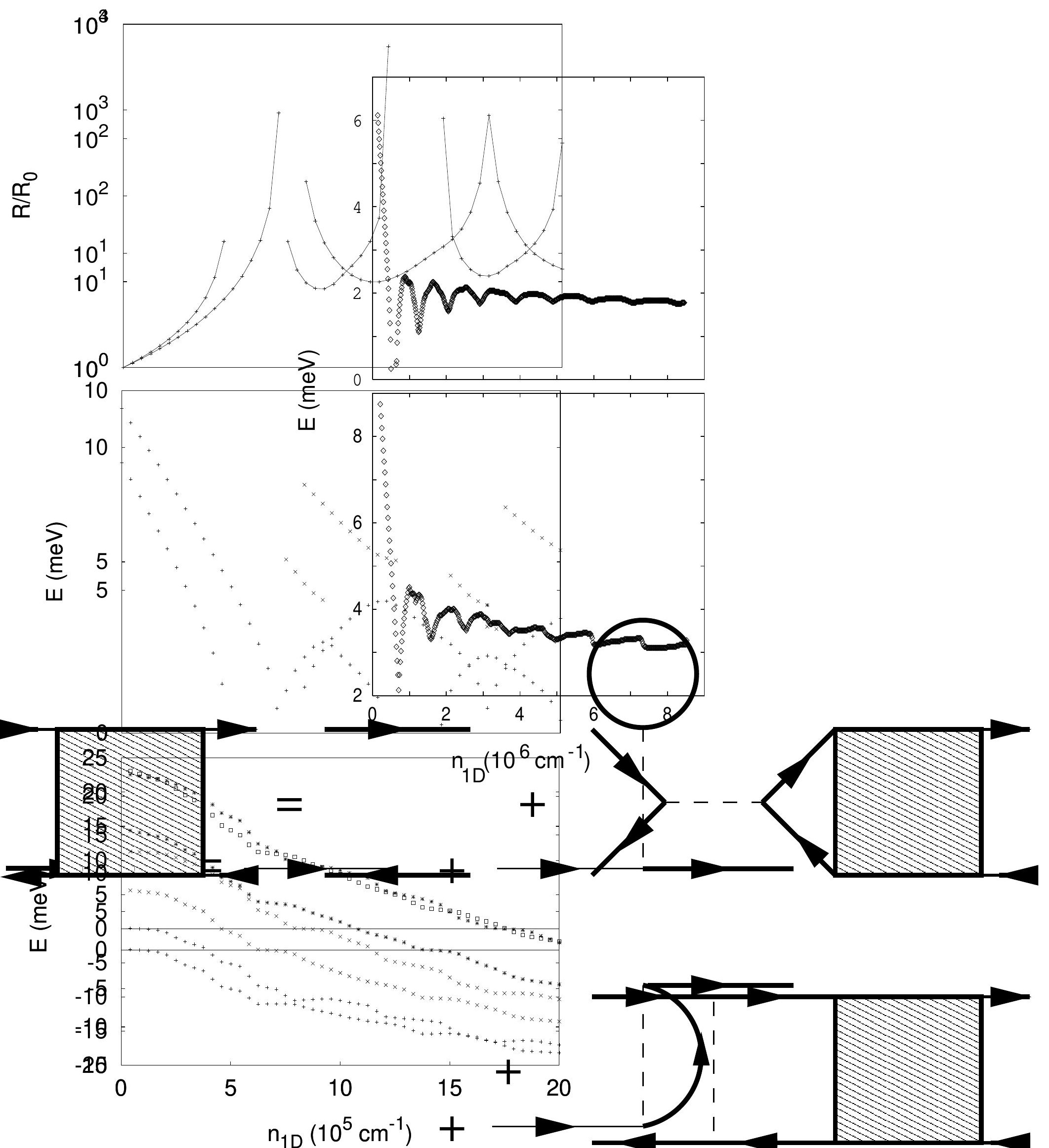




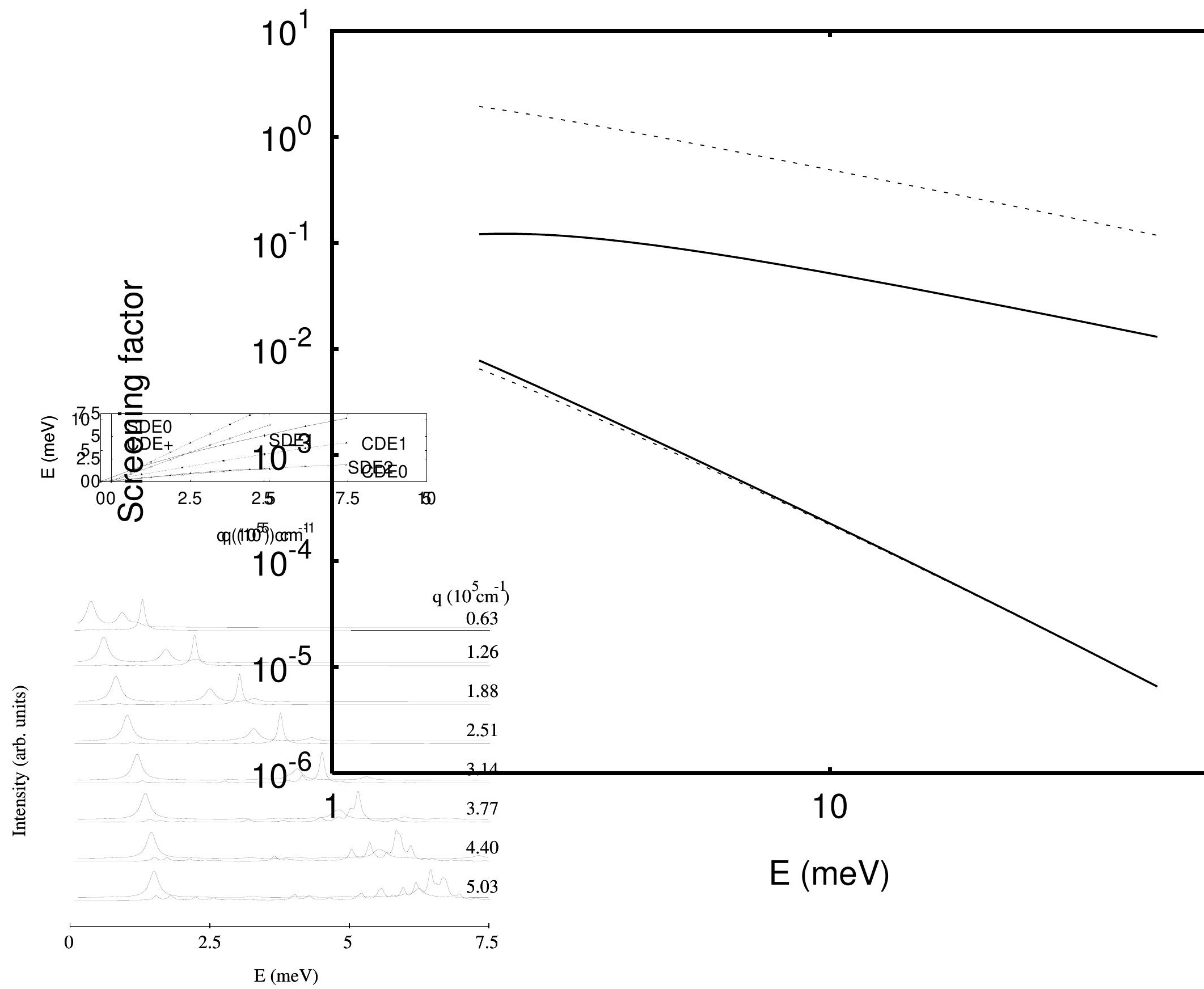

